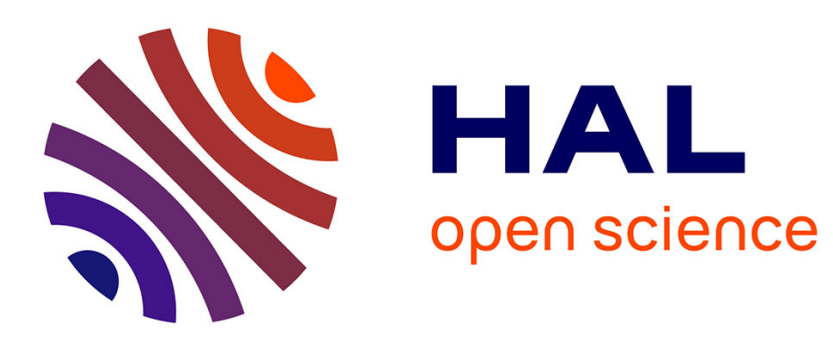

\title{
Facteurs agissant sur la formation des apothécies de Sclerotinia trifoliorum Eriks. en conditions contrôlées
}

Guy Raynal, Francine Ferrari, Michelle Mouret

\section{To cite this version:}

Guy Raynal, Francine Ferrari, Michelle Mouret. Facteurs agissant sur la formation des apothécies de Sclerotinia trifoliorum Eriks. en conditions contrôlées. Agronomie, 1987, 7 (9), pp.715-725. hal00885046

\section{HAL Id: hal-00885046 \\ https://hal.science/hal-00885046}

Submitted on 1 Jan 1987

HAL is a multi-disciplinary open access archive for the deposit and dissemination of scientific research documents, whether they are published or not. The documents may come from teaching and research institutions in France or abroad, or from public or private research centers.
L'archive ouverte pluridisciplinaire HAL, est destinée au dépôt et à la diffusion de documents scientifiques de niveau recherche, publiés ou non, émanant des établissements d'enseignement et de recherche français ou étrangers, des laboratoires publics ou privés. 


\title{
Facteurs agissant sur la formation des apothé- cies de Sclerotinia trifoliorum Eriks. en condi- tions contrôlées
}

\author{
Guy RAYNAL \\ avec la collaboration technique de Francine Ferrari et Michelle Mouret \\ I.N.A.P.G.-I.N.R.A., Laboratoire de Pathologie végétale, Centre de Recherches de Grignon-Massy-Paris, \\ F 78850 Thiverval Grignon
}

Cette étude en conditions contrôlées de la formation des apothécies par S. trifoliorum a pour but d'optimiser les conditions de production d'un inoculum ascosporé pour la sélection de trèfles résistants à la sclérotiniose. Le milieu de production in vitro des sclérotes agit sur la quantité et les dimensions des sclérotes et des apothécies. La taille des sclérotes influe sur la carpogenèse. La dormance des sclérotes peut être levée par dessication à $30^{\circ} \mathrm{C}$ pendant une durée optimale d'un mois. Des sclérotes enfouis dans le sol, à l'extérieur, au printemps, sont aptes à produire des apothécies dès juillet s'ils sont placés en conditions favorables. Enfouis à plus de $2 \mathrm{~cm}$, ils différencient des stipes ramifiés qui n'émergent pas du sol, mais sont aptes à produire des apothécies si on les place à la lumière. La lumière est indispensable à la carpogenèse. Plus l'énergie lumineuse reçue (de 20 à $800 \mu \mathrm{wcm}^{-2}$ ) est forte, plus la différenciation des apothécies est rapide. La lumière blanche est plus favorable, les radiations bleues $(475-500 \mathrm{~nm})$ les moins favorables. L'obscurité continue empêche l'évolution des stipes. La différenciation des apothécies nécessite une forte humidité de l'atmosphère et une imbibition continue du substrat d'incubation des sclérotes. La perlite et le sable sont défavorables à la carpogenèse, contrairement à la vermiculite. Une terre de jardin s'est révélée très favorable, à l'inverse d'un sol limoneux. Enfin, tous les isolats de $S$. trifoliorum de trèfle et de luzerne ne sont pas également aptes à produire des apothécies.

Mots clés additionnels : Carpogenèse, facteurs nutritionnels, facteurs physiques, substrat.

A study under controlled conditions on apothecium formation by $S$. trifoliorum aimed to find the best conditions for the production of ascospores to be used for selection of clovers resistant to crown rot. The culture medium for in vitro production of the sclerotia influenced quantity and dimensions of sclerotia and apothecia. The dimensions of sclerotia had an effect on carpogenesis. The dormancy of sclerotia was removed by drying at $30^{\circ} \mathrm{C}$ for the optimal duration of 1 month. Sclerotia buried outdoors in the soil in spring were able to differentiate apothecia as early as July, when placed in favourable conditions. When the depth of burial exceeded $2 \mathrm{~cm}$, sclerotia differentiated branched stipes which never emerged, but were able to produce apothecia when placed under light. Light was essential to carpogenesis. The higher the light energy (from 20 to $800 \mu \mathrm{wcm}^{-2}$ ), the faster the differentiation of apothecia. White light was most effective, blue waves less. Continuous obscurity inhibited stipe evolution. Apothecium differentiation needed high atmospheric humidity and a damp substrate for the incubation of sclerotia and evolution of stipes. Perlite and sand were unfavourable to carpogenesis, unlike vermiculite. Garden soil was very conducive, but not a silt soil. The S. trifoliorum isolates from clover and lucerne were not all equally able to produce apothecia.

Additional key words : Carpogenesis, nutritional factors, physical factors, substrate.

\section{INTRODUCTION}

Sclerotinia trifoliorum Eriks. est le parasite le plus dommageable du trèfle violet dans les régions tempérées. La sclérotiniose qu'il provoque affecte essentiellement l'installation et la pérennité des cultures et, par là, leur rendement d'une année sur l'autre. En France, cette maladie est vraisemblablement l'un des principaux facteurs qui a conduit les agriculteurs à délaisser le trèfle violet au profit d'autres productions. Rappelons que $S$. trifoliorum entraîne des pourritures hivernales des collets des trèfles qui sont d'autant plus destructrices que les plantes sont plus jeunes. Ainsi, des cultures implantées en fin d'été peuvent être détruites 
au cours de l'hiver suivant pour peu que les conditions soient favorables à la maladie (hiver doux et humide), même pour les variétés considérées actuellement comme ayant un bon niveau de résistance (RAYNAL, 1985).

L'inoculum primaire est constitué par des ascospores se disséminant, pour le Bassin Parisien, de la mioctobre à la mi-novembre environ, à partir des apothécies qui se différencient sur des sclérotes formés au printemps précédent sur et dans les tissus des trèfles que le champignon a tués en hiver. Les ascospores attaquent les folioles en provoquant de nombreuses taches nécrotiques punctiformes qui s'étendent progressivement, sans doute à la faveur de facteurs tels que le froid, qui lèsent plus ou moins les tissus (LOVELESS, 1951 ; DIJKSTRA, 1966b). Le mycélium de ce parasite typiquement nécrotrophe acquiert alors l'énergie nécessaire pour progresser jusqu'aux collets qu'il fait pourrir et pour passer d'une plante à l'autre par simple contact entre organes malades et organes sains, voire en cheminant à la surface du sol.

Des essais de traitements fongicides sont actuellement en cours pour tenter d'enrayer les attaques d'ascospores sur les jeunes cultures. Ils ne peuvent pour l'instant être préconisés au champ, car leurs résultats demandent à être confirmés. La seule méthode de lutte reste donc l'utilisation de cultivars à bon niveau de résistance. On dispose actuellement d'un certain nombre de cultivars de trèfle violet intéressants, notamment parmi les tétraploïdes (RAYNAL, 1985). Cependant, la relance souhaitée de la culture du trèfle violet passe par la sélection de cultivars ayant des rendements accrus et obligatoirement résistants ou très peu sensibles à la sclérotiniose.

La sélection dans les conditions du champ, pratiquée jusque-là, a donné de bons résultats mais s'avère assez aléatoire et très lente. C'est pourquoi nous avons dans un premier temps mis au point diverses techniques applicables en sélection d'évaluation du niveau de résistance des trèfles en chambre de culture (RAYNAL, 1981). Si l'utilisation comme inoculum du mycélium jeune de $S$. trifoliorum produit in vitro est possible, au moins pour classer les sensibilités variétales, l'emploi d'un inoculum ascosporé permet de se rapprocher davantage des conditions naturelles ce qui, a priori, devrait rendre plus fiable la technique de sélection. Ceci impose de produire au laboratoire de grandes quantités d'apothécies sporifères tout au long de l'année. Nous avons déjà exposé les éléments d'une méthode qui permet l'obtention régulière de nombreuses apothécies dans des conditions totalement contrôlées (RAYNAL, 1983). Nous avons depuis effectué de nombreux essais dans le but d'améliorer cette méthode afin de produire de façon programmée un inoculum abondant utilisable à la demande par les sélectionneurs. Ceci nous a conduit en particulier à étudier l'action de divers facteurs sur la carpogenèse et la maturation des apothécies de $S$. trifoliorum. Nous nous proposons ici de faire le point sur ces expérimentations qui ont porté sur plusieurs années et qui nécessitent une synthèse, d'autant plus qu'à notre connaissance notre laboratoire est le premier et pour l'instant le seul dans le monde à produire massivement en conditions contrôlées des apothécies de $S$. trifoliorum en vue de leur utilisation pour la sélection de trèfles violets résistants.

\section{MATÉRIEL ET MÉTHODE GÉNÉRALE}

La définition des conditions optimales pour la production des apothécies n'a pu se faire qu'à la suite de multiples essais plurifactoriels échelonnés dans le temps. Pour clarifier l'analyse des effets des divers facteurs étudiés, nous ferons dans ce qui suit le point sur les résultats obtenus en ne faisant varier qu'un seul facteur à la fois, les autres étant ajustés de façon à être favorables à la carpogenèse. De plus, étant donné la diversité des facteurs analysés, ce chapitre se borne à indiquer la méthode générale utilisée, les méthodes particulières à l'étude de chaque facteur étant exposées en préambule aux résultats correspondants.

Nous avons déjà défini dans leurs grandes lignes les principales conditions favorables à la carpogenèse (RAYNAL, 1984) pour aboutir aux étapes suivantes qui semblent pour l'instant les meilleures pour la production des apothécies tout au long de l'année :

- Utilisation de l'isolat S.T. 78.02 de S. trifoliorum, isolé de trèfle violet à $\mathrm{La}$ Minière (78) en automne 1980 à partir d'apothécies.

- Obtention des sclérotes après 6 semaines de culture du champignon en boîtes de Roux, sur tranches de pomme de terre autoclavées et placées à l'obscurité à $20{ }^{\circ} \mathrm{C}$.

- Récolte des sclérotes, lavage à l'eau du robinet, séchage $24 \mathrm{~h}$ à la température du laboratoire.

- Levée de la dormance des sclérotes par dessication pendant 1 mois à $30^{\circ} \mathrm{C}$, établissement de leur poids sec après ce temps.

- Dépôt des sclérotes, en les enfouissant très légèrement, dans de la vermiculite non stérile maintenue continuellement humide par excès d'eau, contenue dans des bacs de "Miniserres » dont on referme les couvercles pour avoir une humidité maximale. Par «Miniserre », on répartit $50 \mathrm{~g}$ de sclérotes secs.

- Conditions d'incubation des sclérotes : température de $15^{\circ} \mathrm{C}$, lumière fluorescente blanche de $400 \mu \mathrm{wcm}^{-2}$ (environ $1200 \mathrm{lux}$ ), photopériode de $12 \mathrm{~h} / 24 \mathrm{~h}$.

- Première récolte des apothécies 3 semaines après le dépôt des sclérotes sur vermiculite, la récolte se poursuivant pendant 6 à 8 semaines et étant optimale si les conditions de température, d'humidité et d'éclairement demeurent pendant cette période égales à celles citées précédemment.

Dans les essais exposés au chapitre suivant, les conditions de cette méthode sont respectées pour les facteurs fixes, le facteur étudié étant variable. Nous avons ainsi envisagé les facteurs suivants capables d'agir sur la carpogenèse :

- milieu de production des sclérotes,

- grosseur des sclérotes,

- conditions de levée de dormance des sclérotes, le sol

- profondeur d'enfouissement des sclérotes dans

- type de substrat d'incubation des sclérotes,

- facteurs physiques : température, lumière, humidité relative,

- isolats de $S$. trifoliorum.

Enfin, nous ferons état de quelques anomalies observées lors de la formation des apothécies. 


\section{RÉSULTATS ET DISCUSSIONS}

\section{A. Influence du milieu de culture utilisé pour la pro- duction des sclérotes}

Nous avons comparé 4 types de milieux stériles: PDA en boîtes de Petri ( $20 \mathrm{ml} /$ boîte), tranches ou purée de carottes en Erlenmeyers (50 g/Erlen), tranches de pommes de terre (cultivar Bintje) réparties en boîtes de Roux ( $200 \mathrm{~g} /$ boîte). Le champignon est repiqué avec des implants mycéliens prélevés à la périphérie de cultures de 3 jours obtenues sur PDA à $20^{\circ} \mathrm{C}$. Un implant est nécessaire par boîte de Petri et par Erlenmeyer, alors qu'il en faut 2 par boîte de Roux.

Après observation des autres conditions citées dans le chapitre II, la production des sclérotes, des apothécies et des ascospores s'établit comme indiqué dans le tableau 1. La récolte des ascospores est réalisée en laissant sécher à l'air libre les apothécies détachées de leur stipe et dont l'hyménium est plaqué contre un fond de boîte de Petri (Tourvielle De Labroune $e t$ al., 1978). Après mise en suspension dans de l'eau, les ascospores sont dénombrées à l'hématimètre en groupant des lots de 100 apothécies.

Les résultats indiqués pour la production de sclérotes et d'apothécies sont des moyennes établies à la suite de nombreuses manipulations échelonnées dans le temps, sur au total quelque 400 boîtes de Petri, 500 boîtes Roux et 250 Erlenmeyers. De même, les dimensions des apothécies âgées de 4 à 5 jours et la quantité d'ascospores produites par apothécie à cet âge sont évaluées sur 1 essai à partir de 150 apothécies seulement dans le cas des milieux à base de PDA et de carottes, et sur plusieurs milliers pour les pommes de terre en tranches (nombreux essais).

On ne peut donc en toute rigueur comparer statistiquement ces résultats entre eux de façon globale. Nous considérons cependant que ces résultats sont sûrs, étant donné le grand nombre d'observations concordantes auxquelles ils ont donné lieu.
Ainsi que nous l'avions déjà noté (RAYNAL, 1983), bien que les sclérotes produits sur milieu à base de carotte ne diffèrent pas par leur aspect de ceux produits sur milieux à base de pomme de terre (PDA et tranches), ils sont nettement moins productifs en apothécies. Dans tous les cas, le rendement en sclérotes, en apothécies et finalement en ascospores est le plus élevé lorsque les sclérotes sont obtenus sur tranches de pomme de terre en boîtes de Roux. Remarquons la variabilité élevée des résultats qui conduit, même dans les conditions apparemment les plus favorables, à des fluctuations pouvant atteindre 30 à 50 p. 100 d'un essai à l'autre.

Le diamètre moyen des apothécies dépend des milieux de production des sclérotes, mais aussi de leur âge. Ainsi, les diamètres rapportés dans le tableau 1 et par la suite sont établis à partir d'apothécies âgées de 4 à 5 jours (intervalle entre 2 récoltes) dont l'hyménium est étalé. $\mathrm{Si}$ on laisse les apothécies atteindre leur taille maximale (à $7-15$ jours), leur diamètre moyen sur sclérotes de tranches de pomme de terre s'établit à $6,75 \pm 1,21 \mathrm{~mm}$ (sur 100 apothécies), les plus grosses atteignant $9,50 \mathrm{~mm}$. Le rendement en ascospores est alors accru. De plus, notre mode de récolte des ascospores est loin de permettre d'obtenir la totalité des ascospores qu'une apothécie est capable de produire pendant toute sa vie, qui peut atteindre 2 à 3 semaines. Des manipulations en cours nous montrent en effet qu'une apothécie peut projeter, en conditions favorables, jusqu'à 3 à $4 \times 10^{6}$ ascospores en 3 semaines. Nous sommes proches de la valeur moyenne relevée par SCHWARTZ \& STEAMAN (1978) avec $S$. sclerotiorum sur un très petit nombre d'apothécies, de $2,32 \times 10^{6}$ ascospores représentant la décharge d'une apothécie pendant 9 jours au laboratoire. La technique de récolte des ascospores que nous utilisons nous semble cependant la plus appropriée pour l'obtention d'une grande quantité d'inoculum facile à conserver, pour la sélection de la résistance du trèfle violet.

Enfin, la maîtrise des facteurs conduisant à la ger-

TABLEAU 1

Influence du milieu de production des sclérotes sur la carpogenèse.

Influence on carpogenesis of the medium used for the production of sclerotia.

\begin{tabular}{|c|c|c|c|c|c|c|c|}
\hline Milieu & $\begin{array}{c}\text { Poids sec } \\
\text { moyen }(\mathrm{g}) \\
\text { de sclérotes } \\
\text { par récipient }\left(^{*}\right) \\
\pm \text { écart type }\end{array}$ & $\begin{array}{l}\text { Poids sec } \\
\text { moyen }(\mathrm{g}) \\
\text { de } 100 \\
\text { sclérotes } \\
\pm \text { écart type }\end{array}$ & $\begin{array}{l}\text { Nombre moyen } \\
\text { d'apothécies } \\
\text { pour } 100 \\
\text { sclérotes } \\
\pm \text { écart type }\end{array}$ & $\begin{array}{l}\text { Nombre moyen } \\
\text { d'ascospores } \\
\text { récolté } \\
\text { par apothécie } \\
\pm \text { écart type }\end{array}$ & $\begin{array}{c}\varnothing \text { moyen } \\
\left(10^{-1} \mathrm{~mm}\right) \\
\text { d'une } \\
\text { apothécie } \\
\pm \text { écart type }\end{array}$ & $\begin{array}{l}\text { Rendement } \\
\text { approché } \\
\text { en ascospores } \\
\text { par type } \\
\text { de récipient }\end{array}$ & $\begin{array}{l}\text { Rendement } \\
\text { approché } \\
\text { en ascospores } \\
\text { par g de milieu } \\
\text { de culture }\end{array}$ \\
\hline PDA $\left({ }^{*}\right): 1$ & $0,43 \pm 0,28$ & $0,42 \pm 0,26$ & $32,6 \pm 12,8$ & $45000 \pm 10300$ & $33,9 \pm 6,9$ & $1,5 \times 10^{6}$ & $75 \times 10^{3}$ \\
\hline $\begin{array}{l}\text { Carottes } \\
\text { en tranches } \\
\left({ }^{*}\right): 2\end{array}$ & $2,01 \pm 0,43$ & $5,33 \pm 3,89$ & $10,4 \pm 4,3$ & $52500 \pm 22800$ & $47,2 \pm 10,9$ & $0,2 \times 10^{6}$ & $4 \times 10^{3}$ \\
\hline $\begin{array}{c}\text { Carottes } \\
\text { en purée } \\
\left({ }^{*}\right): 2\end{array}$ & $2,15 \pm 0,58$ & $5,24 \pm 2,91$ & $23,1 \pm 9,6$ & $40200 \pm 24000$ & $40,2 \pm 8,4$ & $0,4 \times 10^{6}$ & $8 \times 10^{3}$ \\
\hline $\begin{array}{c}\text { Pommes } \\
\text { de terre en } \\
\text { tranches }\left({ }^{*}\right): 3\end{array}$ & $27,84 \pm 3,58$ & $2,54 \pm 0,87$ & $54,7 \pm 20,5$ & $86000 \pm 37000$ & $51,1 \pm 10,6$ & $48 \times 10^{6}$ & $240 \times 10^{3}$ \\
\hline
\end{tabular}

$\left(^{*}\right)$ Type de récipients : 1 : boîte de Petri ; 2 : Erlenmeyer ; 3 : boîte de Roux. 
mination carpogénique des sclérotes est loin d'être parfaite, puisqu'ils ne produisent pas tous d'apothécies.

\section{B. Influence de la grosseur des sclérotes}

Les sclérotes récoltés sur PDA sont en moyenne petits et de dimensions homogènes lorsqu'ils sont secs (dimensions $<3 \mathrm{~mm}$ ). Par contre, sur milieux à base de carotte ou sur tranches de pomme de terre, on a toute une gamme de dimensions de sclérotes, dont la taille peut atteindre à l'état sec jusqu'à 15-20 mm. Nous avions déjà constaté (RAYNAL, 1983) une relation entre la taille des sclérotes et le nombre d'apothécies qu'ils produisent. Nous quantifions ici précisément cette relation.

Pour ce faire, nous utilisons 2 récoltes de sclérotes, en 2 essais, produits sur tranches de pomme de terre. Après tamisage, les sclérotes secs sont répartis en 4 lots selon leur taille. Les lots sont placés en conditions favorables pour la production des apothécies, lesquelles sont récoltées pendant 1 mois.

Le tableau 2 montre là encore une forte variabilité entre les 2 essais, ce qui nous a obligé à ne pas les assembler. Cependant, dans les 2 cas, les sclérotes petits $(<3 \mathrm{~mm})$ et moyens $(3-6 \mathrm{~mm})$ sont les plus abondants et produisent corrélativement la plus grande quantité d'apothécies par rapport au total. Par contre, plus la taille des sclérotes augmente, plus le nombre moyen d'apothécies par sclérote progresse en général. En effet, les petits sclérotes ne donnent naissance la plupart du temps qu'à une seule apothécie, alors que les plus gros ( $>10 \mathrm{~mm}$ ) peuvent en porter plusieurs en même temps (jusqu'à une vingtaine pour les très gros). Ces gros sclérotes sont cependant loin de produire tous des apothécies, alors que les petits sont facilement plus fructifères, comme si la levée de dormance des sclérotes se faisait de façon plus homogène pour ceux dont les dimensions sont réduites.

\section{Influence des conditions de levée de dormance des sclérotes}

\section{Levée de dormance par dessication à $30^{\circ} \mathrm{C}$}

Dans la nature, les sclérotes restent généralement en repos depuis leur formation (février-avril) jusqu'à l'automne suivant où ils forment des apothécies (RAYNAL, 1983, 1985 ; WILLIAMS \& WESTERN, 1965a). Au laboratoire, on peut lever la dormance des sclérotes par divers traitements chimiques et thermiques (Dijkstra, 1966a, MCGimpsey \& MAlone, 1979). Nous avons montré que la dessication à la température du laboratoire pendant 1 à 6 mois, suivie de 24 heures de congélation, de sclérotes produits sur PDA et carottes, permet d'améliorer considérablement la production d'apothécies (RAYNAL, 1983). Diverses manipulations nous ont conduit depuis, avec les sclérotes produits sur tranches de pomme de terre, à préférer une température constante de dessication de $30{ }^{\circ} \mathrm{C}$, sans congélation ultérieure, avant de transférer les sclérotes sur la vermiculite humide. L'établissement de la durée optimale de dessication a fait l'objet

TABLEAU 2

Effet de la dimension des sclérotes sur la quantité et le diamètre d'apothécies produites pendant I mois (2 essais, a et b). Influence of the dimensions of sclerotia on the quantity and diameter of apothecia produced during 1 month (2 experiments, a and b).

\begin{tabular}{|c|c|c|c|c|c|c|c|}
\hline $\begin{array}{l}\text { Dimensions } \\
\text { des sclérotes } \\
\text { secs }\end{array}$ & Essais & $\begin{array}{l}\text { Poids sec (g) } \\
\text { et pourcentage } \\
\text { de sclérotes }\end{array}$ & $\begin{array}{l}\text { Nombre et } \\
\text { pourcentage } \\
\text { de sclérotes }\end{array}$ & $\begin{array}{l}\text { Nombre total } \\
\text { et pourcentage } \\
\text { d'apothécies }\end{array}$ & $\begin{array}{l}\text { Nombre } \\
\text { d'apothécies } \\
\text { par gramme } \\
\text { de sclérotes }\end{array}$ & $\begin{array}{c}\text { Nombre } \\
\text { d'apothécies } \\
\text { pour } 100 \\
\text { sclérotes }\end{array}$ & $\begin{array}{c}\text { Diamètre } \\
\text { des apothécies } \\
(\mathrm{mm})\end{array}$ \\
\hline \multirow{2}{*}{$<3 \mathrm{~mm}$} & a & $\begin{array}{c}39 \\
6,0 \%\end{array}$ & $\begin{array}{r}8200 \\
33,5 \quad \%\end{array}$ & $\begin{array}{r}3519 \\
20,3 \%\end{array}$ & 92,6 & 43 & $3,60 \pm 0,77$ \\
\hline & $b$ & $\frac{52}{12,0 \%}$ & $\begin{array}{l}11600 \\
62,8 \%\end{array}$ & $\begin{array}{r}7309 \\
35,3 \%\end{array}$ & 140,5 & 63 & $4,10 \pm 0,45$ \\
\hline \multirow{2}{*}{$3-6 \mathrm{~mm}$} & $\mathrm{a}$ & $\begin{array}{c}437 \\
68,9 \%\end{array}$ & $\begin{array}{l}15000^{*} \\
61,3 \%\end{array}$ & $\begin{array}{r}12828 \\
74,0 \%\end{array}$ & 29,3 & 85 & $4,38 \pm 0,80$ \\
\hline & $\mathrm{b}$ & $\begin{array}{c}230 \\
53,0 \%\end{array}$ & $\begin{array}{r}6000 \\
32,5 \%\end{array}$ & $\begin{array}{r}9371 \\
45,3 \%\end{array}$ & 40,7 & 156 & $5,10 \pm 0,82$ \\
\hline \multirow{2}{*}{$6-10 \mathrm{~mm}$} & $\mathrm{a}$ & $\begin{array}{c}140 \\
22,1 \%\end{array}$ & $\begin{array}{l}1200 \\
4,9 \%\end{array}$ & $\begin{array}{r}848 \\
4,9 \%\end{array}$ & 6,1 & 71 & $5,10 \pm 0,79$ \\
\hline & $b$ & $\begin{array}{c}98 \\
22,6^{\circ \%}\end{array}$ & $\begin{array}{r}700 \\
3,8 \%\end{array}$ & $\begin{array}{r}2714 \\
13,1 \%\end{array}$ & 27,6 & 388 & $4,58 \pm 0,63$ \\
\hline \multirow{2}{*}{$>10 \mathrm{~mm}$} & a & $\begin{array}{c}19 \\
3,0 \%\end{array}$ & $\begin{array}{c}70 \\
0,3^{\%}\end{array}$ & $\begin{array}{r}131 \\
0,8 \%\end{array}$ & 6,9 & 187 & $4,87 \pm 1,15$ \\
\hline & $\mathrm{b}$ & $\begin{array}{c}54 \\
12,4 \%\end{array}$ & $\begin{array}{r}160 \\
0,9 \%\end{array}$ & $\begin{array}{l}1283 \\
6,3 \%\end{array}$ & 23,7 & 802 & $3,94 \pm 1,12$ \\
\hline \multirow{2}{*}{$\begin{array}{l}\text { Total ou } \\
\text { moyenne* }\end{array}$} & $\mathrm{a}$ & 634 & 24470 & 17326 & $32,6^{*}$ & $70^{*}$ & $4,49^{*}$ \\
\hline & b & 434 & 18460 & 20677 & $47,6^{*}$ & $112^{*}$ & $4,43^{*}$ \\
\hline
\end{tabular}


de plusieurs essais, que nous résumons dans le tableau 3.

\section{TABLEAU 3}

Influence de la durée de dessication des sclérotes à $30{ }^{\circ} \mathrm{C}$ sur la production d'apothécies $(50 \mathrm{~g}$ soit environ 800 sclérotes produits sur tranches de pomme de terre par durée).

Influence of time of desiccation of sclerotia at $30{ }^{\circ} \mathrm{C}$ on the production of apothecia $150 \mathrm{~g}$ i.e. about 800 sclerotia produced on potato slices for each duration).

\begin{tabular}{ccc}
\hline $\begin{array}{c}\text { Durée de } \\
\text { dessication } \\
\text { (semaines) }\end{array}$ & $\begin{array}{c}\text { Nombre moyen } \\
\text { d'apothécies pour } \\
\text { 100 sclérotes }\end{array}$ & $\begin{array}{c}\text { Durée de } \\
\text { production } \\
\text { (semaines) }\end{array}$ \\
\hline 0 & 2,4 & 6 \\
1 & 17,7 & 7 \\
2 & 36,6 & 7 \\
4 & 58,1 & 7 \\
8 & 3,6 & 5 \\
\hline \hline
\end{tabular}

La durée optimale de dessication, dans nos conditions, s'établit à 1 mois. Elle lève la dormance du maximum de sclérotes possible. Une température élevée, alliée à la sécheresse, semble avoir un effet bénéfique sur la carpogenèse, puisque les sclérotes produisent d'autant plus d'apothécies qu'ils subissent plus ces conditions, jusqu'à un optimum. Au-delà de 1 mois, la viabilité des sclérotes est affectée, ce qui retentit sur la formation des apothécies. On peut donc penser que dans la nature, les conditions climatiques estivales constituent un des facteurs de levée de la dormance des sclérotes. La dessication à $30^{\circ} \mathrm{C}$ pendant 1 mois ne lève pas la dormance de tous les sclérotes, car plus de la moitié ne produisent pas d'apothécies. Contrairement à ceux qui ont différencié des stipes et des apothécies, et qui se dégradent plus ou moins vite après avoir fructifié, la plupart des sclérotes stériles demeurent fermes, bien qu'imbibés, avec une région médullaire blanche ou blanc grisâtre, même après avoir passé 2 mois sur la vermiculite humide. Après lavage et une nouvelle dessication, une proportion de ces sclérotes, très variable d'un essai à l'autre, est capable de former des apothécies. Cette detrxième récolte est peu utilisable, car peu abondante et sujette à des infections fréquentes par Gliocladium roseum (Bain.) qui fructifie sous forme d'une farine blancrosé sur les organes atteints qui pourrissent.

\section{Levée de dormance par les conditions naturelles}

Le passage à l'étuve des sclérotes pendant 1 mois est loin de refléter les conditions du champ. Pour mieux comprendre le phénomène de levée de dormance dans les conditions naturelles, nous avons, dès leur récolte à partir de tranches de pomme de terre, réparti les sclérotes dans des sachets de toile à bluter en nylon, qui furent aussitôt enfouis au champ à $10 \mathrm{~cm}$ de profondeur le 18 mars 1983 , soit à l'époque de formation des sclérotes dans la nature. Cette technique est employée par LAMARQUE (1980) pour obtenir des apothécies de $S$. sclerotiorum, après 4 mois de séjour dans le sol, à partir de sclérotes produits au laboratoire. Dès le 6 juin 1983, puis à des dates échelonnées. nous avons déterré les sachets et placé les sclérotes à $15^{\circ} \mathrm{C}$ sur vermiculite humide afin de voir, dès lors qu'ils produisent des apothécies, à quel moment leur dormance est levée. Le tableau 4 donne les résultats de cet essai. La levée de dormance n'est effective au plus tôt qu'entre le 22 juin et le 22 juillet. D'après le tableau 4, elle serait maximale pour les sclérotes déterrés le 22 août. En fait, si la production d'apothécies diminue fortement pour les sclérotes déterrés à partir du 20 septembre, ce n'est pas qu'ils soient à nouveau inhibés dans leur germination carpogénique, mais parce qu'ils sont en très mauvais état. Ils sont en effet plus ou moins pourris en grand nombre, et donnent naissance à des apothécies et des stipes qui noircissent rapidement, à cause du parasitisme par Gliocladium roseum.

$\mathrm{Si}$ dans la nature, les sclérotes ne forment pas d'apothécies en été, du moins dans la moitié nord de la France, bien que leur dormance soit vraisemblablement levée, c'est donc que les températures estivales s'opposent à la différenciation des apothécies. Il pourrait en être autrement dans les pays nordiques. Nous verrons en effet que les températures élevées sont défavorables à la formation et à l'évolution des apothécies. D'autre part, les sclérotes prélevés à partir du 20 octobre montrent à leur sortie de terre des stipes plus ou moins tortueux qui n'ont pas évolué en apothécies et qui sont en voie de nécrose. Etant profondément enfouis dans le sol et emprisonnés dans les sachets de toile, ils n'ont pu émerger et n'ont donc pas fructifié. Ils étaient pourtant aptes à produire des apothécies au champ à partir de cette époque, ce qui recoupe toutes nos précédentes observations publiées ou non sur la formation des apothécies au champ ou en conditions semi-naturelles (RAYNAL, 1983, 1985).

TABI.EAU 4

Influence des conditions naturelles sur la levée de dormance de sclérotes enfouis dans le sol le 18 mars 1983 (voir texte). Influence of natural conditions on dormancy removal of sclerotia buried in the soil on 18 March 1983 (see text).

\begin{tabular}{|c|c|c|c|c|c|}
\hline \multirow{2}{*}{$\begin{array}{c}\text { Date de } \\
\text { déterrement } \\
\text { des sclérotes (1983) }\end{array}$} & \multirow{2}{*}{$\begin{array}{l}\text { Nombre } \\
\text { de mois } \\
\text { en terre }\end{array}$} & \multicolumn{2}{|c|}{ Production d'apothécies } & \multirow{2}{*}{$\begin{array}{l}\text { Délai entre date de } \\
\text { déterrement et début de } \\
\text { production des apothécies }\end{array}$} & \multirow{2}{*}{$\begin{array}{l}\text { Nombre } \\
\text { d'apothécies pour } \\
100 \text { sclérotes }\end{array}$} \\
\hline & & Début & Fin & & \\
\hline 6 juin & 2,5 & \multicolumn{2}{|c|}{ aucune production } & - & 0 \\
\hline 22 juin & 3 & \multicolumn{2}{|c|}{ aucune production } & - & 0 \\
\hline 22 juillet & 4 & 12 août & 21 septembre & $21 \mathrm{j}$ & 23,5 \\
\hline 22 août & 5 & 9 septembre & 10 octobre & $17 \mathrm{j}$ & 71,0 \\
\hline 20 octobre & 7 & 7 novembre & 4 janvier & $18 \mathrm{j}$ & 24,4 \\
\hline 21 novembre & 8 & 5 décembre & 4 janvier & $14 \mathrm{j}$ & 1,2 \\
\hline
\end{tabular}




\section{Effet de la profondeur d'enfouissement des sclérotes}

Nous venons de voir que des sclérotes enfouis suffisamment profondément dans le sol sont incapables de différencier des apothécies à l'air libre, bien qu'ils forment des stipes. Il convient cependant de préciser à partir de quelle profondeur les sclérotes sont incapables d'émettre des apothécies et si leurs stipes seraient aptes à différencier des apothécies s'ils retrouvaient des conditions favorables. Nous avons pour cela étudié le devenir de sclérotes enfouis à diverses profondeurs, leur dormance ayant été préalablement levée. Ces sclérotes, de dimensions de 3 à $10 \mathrm{~mm}$ à l'état $\mathrm{sec}$, récoltés sur tranches de pomme de terre et ayant séjourné à $30^{\circ} \mathrm{C}$ pendant 1 mois, sont enfouis en pots de $30 \mathrm{~cm}$ de diamètre dans de la terre de jardin non stérile et maintenue humide, à des profondeurs allant de 0 à $10 \mathrm{~cm}(20 \mathrm{~g}$ de sclérotes par profondeur). Les pots sont placés à $15^{\circ} \mathrm{C}$ sous lumière fluorescente blanche.

\section{TABLEAU 5}

Effet de la profondeur d'enfouissement des sclérotes dans le sol sur la différenciation des stipes et des apothécies.

Influence of depth of burial of sclerotia in the soil on the differentiation of stipes and apothecia.

\begin{tabular}{|c|c|c|}
\hline $\begin{array}{c}\text { Profondeur } \\
\text { d'enfouissement } \\
\text { (cm) }\end{array}$ & $\begin{array}{c}\text { Nombre total } \\
\text { d'apothécies } \\
\text { pour } \\
100 \text { sclérotes } \\
\text { formées } \\
\text { en } 2 \text { mois }\end{array}$ & $\begin{array}{c}\text { Caractères, } 2 \text { mois après } \\
\text { l'enfouissement des sclérotes, } \\
\text { des stipes n'ayant pas donné } \\
\text { d'apothécies }\end{array}$ \\
\hline 0 & 57 & $\begin{array}{l}\text { courts }(<10 \mathrm{~mm}) \text {, droits, } \\
\text { dressés, nombreux }\end{array}$ \\
\hline 1 & 34 & $\begin{array}{l}\text { courts }(<10 \mathrm{~mm}) \text {, plus ou } \\
\text { moins ramifiés, nombreux }\end{array}$ \\
\hline 2 & 5 & $\begin{array}{l}\text { longs (jusqu'à } 20 \mathrm{~mm} \text { ), rami- } \\
\text { fiés, nombreux, noircissant }\end{array}$ \\
\hline 3 à 10 & 0 & $\begin{array}{l}\text { longs (jusqu'à } 40 \mathrm{~mm} \text { ), très } \\
\text { ramifiés, fragiles, nombreux, } \\
\text { noirs }\end{array}$ \\
\hline
\end{tabular}

Les résultats de cette manipulation sont indiqués dans le tableau 5. Pour les sclérotes placés à la surface de la terre ou à $1 \mathrm{~cm}$ de profondeur, les premières apothécies apparaissent après 30 à 35 jours. Pour les sclérotes enterrés à plus de $3 \mathrm{~cm}$, aucune apothécie n'émerge, même après 2 mois, bien que des stipes nombreux, ramifiés et très fragiles soient observables après que les sclérotes aient été déterrés et lavés. Plus les sclérotes sont enfouis profondément, plus les stipes sont longs et ont tendance à noircir (fig. 1). Une forte proportion de sclérotes (plus de $70 \mathrm{p}$. 100) présente ce phénomène. Lorsque les sclérotes porteurs de tels stipes ramifiés sont placés à la lumière à $15^{\circ} \mathrm{C}$ sur coton humide, les extrémités de leurs ramifications différencient de nombreuses apothécies (fig. 2). La lumière est donc absolument nécessaire pour l'évolution normale des stipes, ainsi que nous le verrons plus loin. De plus, lorsque les sclérotes sont enfouis trop profondément, leurs réserves sont vraisemblablement insuffisantes pour permettre aux stipes de croître assez pour émerger à la surface du sol et s'épanouir en apothécies. En outre, plus les sclérotes sont enterrés profondément, plus les stipes sont atteints de nécroses, peutêtre dues à des conditions asphyxiantes. Leurs chances

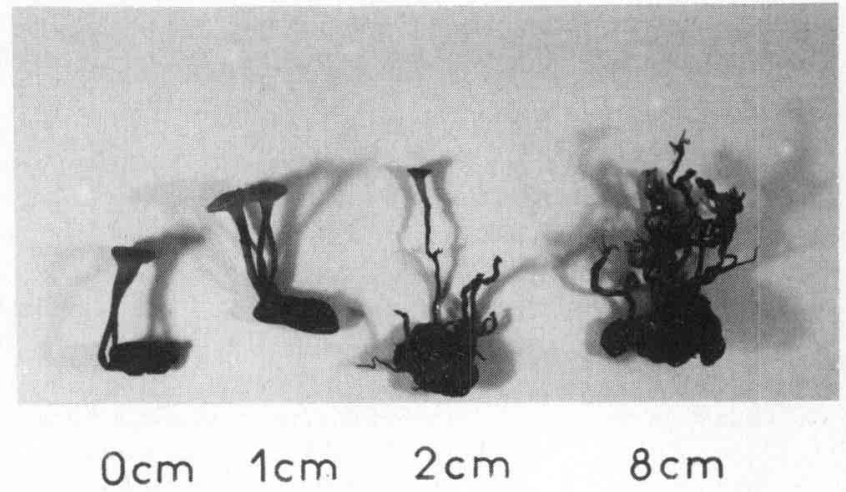

Figure 1

Formation des stipes et des apothécies en fonction de la profondeur d'enfouissement des sclérotes dans le sol $(0$ à $8 \mathrm{~cm})$.

Formation of stipes and apothecia in relation to depth of burial of sclerotia in the soil $(0$ to $8 \mathrm{~cm}$.

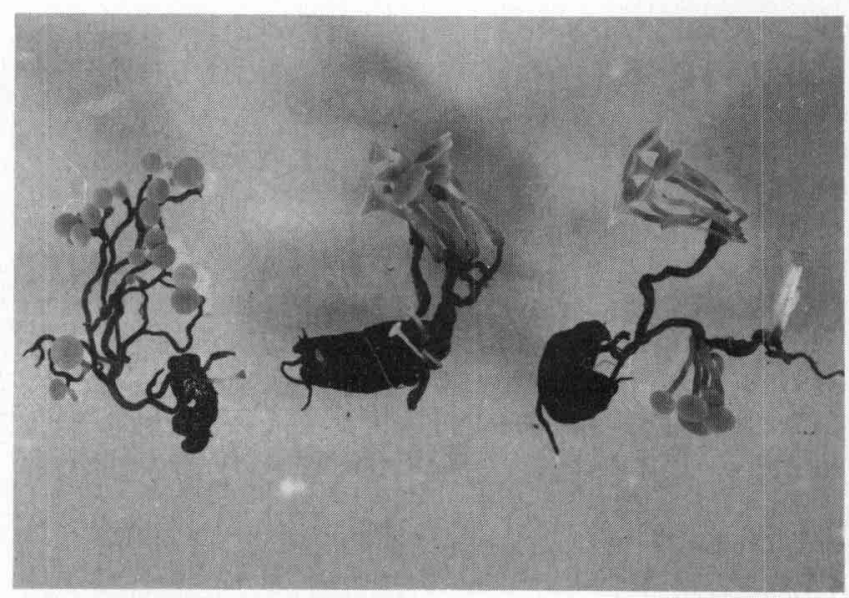

Figure 2

Différenciation d'apothécies sur des stipes ramifiés.

Differentiation of apothecia from branched stipes.

de former des apothécies sont donc pratiquement nulles s'ils restent en profondeur.

11 n'est pas possible de déceler des différences quantitatives de formation des apothécies par les stipes ramifiés, en fonction de la profondeur d'enfouissement des sclérotes. Sur un total de 193 sclérotes examinés porteurs de stipes ramifiés (1 228 stipes au total), 345 apothécies sont apparues après exposition de ces sclérotes à la lumière, correspondant à un pourcentage d'évolution des stipes de 30,6 p. 100. La quantité d'apothécies pour 100 sclérotes fructifères s'élève à 178,7 valeur très semblable à celle que l'on observe pour les sclérotes fructifères montrant des stipes normaux, légèrement enfouis dans la vermiculite. D'autre part, le diamètre des apothécies âgées de 7 à 8 jours produites par les stipes ramifiés ne dépasse pas $3 \mathrm{~mm}$, si bien qu'au total, le rendement en ascospores de ces sclérotes est relativement faible.

Remarquons que WILLIAMS \& WESTERN (1965a) avaient également observé pour des sclérotes de $S$. $t r i$ foliorum et $S$. sclerotiorum enfouis au champ à diverses profondeurs du printemps à l'été, que la formation des apothécies (été-automne pour $S$. sclerotiorum, automne seulement pour $S$. trifoliorum) n'avait 
lieu que pour les sclérotes enterrés le plus superficiellement. Ils émettaient cependant l'hypothèse (1965b) que le labour pourrait ramener à la surface du sol des sclérotes viables qui seraient capables de former des apothécies, d'où l'explication d'attaques de jeunes cultures de trèfles dans des sols où une longue rotation a pourtant été effectuée. Au vu de nos essais en chambre de culture, il nous semble peu probable que des labours d'automne - époque dans la nature de la différenciation des stipes et des apothécies de $S$. trifoliorum - puissent ramener intacts à la surface du sol, des sclérotes enfouis profondément qui présenteraient des stipes ramifiés. Seuls les labours de printemps ou de fin d'été, avant le semis du trèfle violet, pourraient éventuellement remonter des sclérotes enfouis jusqu'aux horizons superficiels, leur permettant alors de fructifier normalement. Il nous semble in fine qu'en règle générale, le retournement par des labours profonds d'automne des parcelles très attaquées de trèfle violet, devrait aboutir, ainsi qu'on le pense ordinairement, à inactiver par enfouissement la majorité des sclérotes. Il resterait à savoir si la profondeur d'enfouissement joue par elle-même sur la précocité et l'intensité de la levée de dormance des sclérotes, en enfouissant à diverses profondeurs des sclérotes venant d'être récoltés et, comme nous l'avons fait au $\S \mathrm{C}$, en les reportant à des dates échelonnées dans des conditions favorables.

\section{E. Effet du substrat d'incubation des sclérotes}

Pour des raisons de commodité, nous utilisons de la vermiculite humidifiée comme substrat d'incubation des sclérotes. On peut envisager d'autres supports faciles à humidifier et peu coûteux, par exemple de la terre, du sable, de la perlite. Nous avons comparé ces divers substrats, stérilisés ou non à l'autoclave. Deux types de sols sont utilisés : terre calcaire de jardin bien équilibrée et sol limoneux (horizon de surface de parcelles de trèfle violet de la Station I.N.R.A. de La Minière (78) où l'on trouve aisément des apothécies de S. trifoliorum en automne). Chaque substrat est testé en 4 "Miniserres" à raison de $50 \mathrm{~g}$ de sclérotes par bac dans les conditions citées au chapitre II. Les caractéristiques de la production des apothécies sur 1 mois, observée 1 mois après le dépôt des sclérotes sur le substrat, sont données dans le tableau 6.

Cet essai, comme tous ceux sur $S$. trifoliorum, montre une variabilité élevée. Néanmoins, on voit nettement l'influence de facteurs physico-chimiques et peut-être biologiques dans le cas du sol sur la différenciation des apothécies.

Ainsi, la terre de jardin constitue le substrat le plus favorable, particulièrement lorsqu'elle n'est pas stérilisée (fig. 3). La production des apothécies y est 3 à 4 fois plus abondante que sur la vermiculite, considérée comme substrat de référence. Cependant, l'emploi de terre, stérilisée ou non, conduit à la longue plus facilement au développement de Gliocladium roseum qui devient envahissant en se répandant par ses spores dans la chambre de culture, ce qui gène considérablement les manipulations ultérieures. L'utilisation de terre n'est donc pas conseillée pour une production continuelle d'apothécies.

Contrairement à ce que l'on pourrait attendre, le sol de La Minière n'est pas favorable dans nos conditions à la différenciation des apothécies. La différence avec ce qui se passe au champ peut s'expliquer par le fait que dans les parcelles, les apothécies se forment, non pas sur le sol libre, mais sur les rangs de trèfles, aux

TABLEAU 6

Influence du substrat sur la production d'apothécies (durée de production observée : 1 mois).

Influence of the substrate on the production of apothecia (production observed during 1 month).

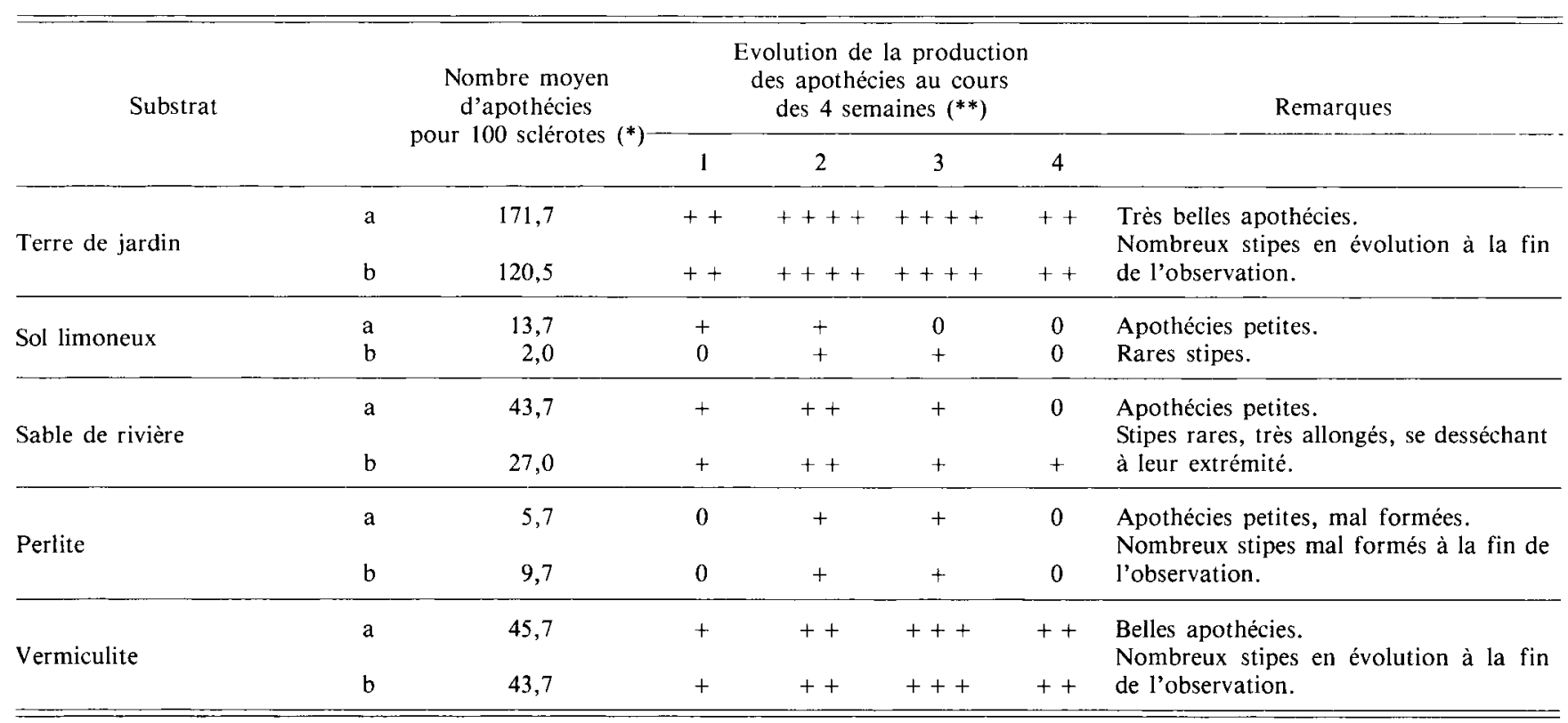

a : substrat non stérile.

b : substrat stérilisé à l'autoclave.

$\left({ }^{*}\right)$ : P.p.d.s. $5 \%=18,5$; c.v. $=26,3 \%$.

$\left({ }^{* *}\right): 0=$ aucune apothécie. Le nombre de signes + exprime l'intensité de production des apothécies. 


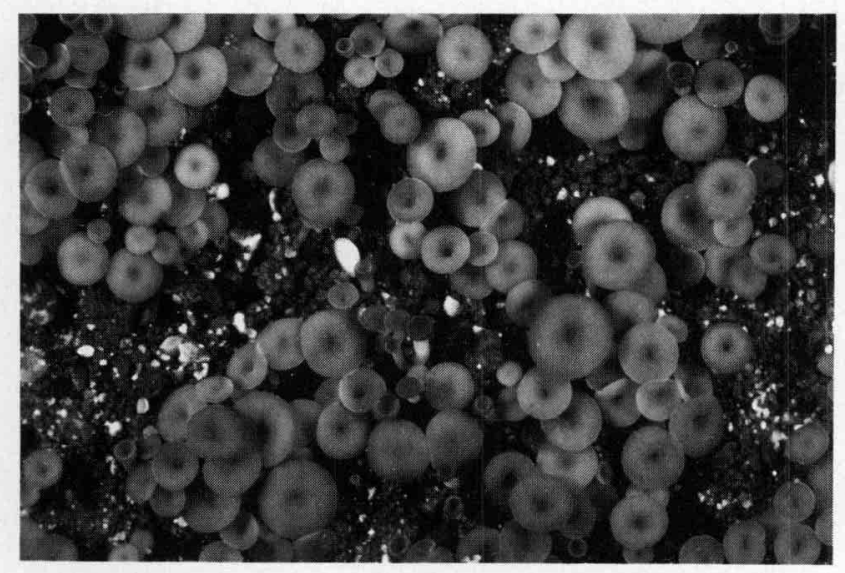

Figure 3

Production d'apothécies sur un substrat de terre de jardin.

Production of apothecia on a garden soil substrate.

emplacements des pieds morts. On peut donc imaginer que les sclérotes immergés dans les débris de végétation ne sont pas en contact direct avec le sol qui, de ce fait, influe moins ou pas du tout sur la carpogenèse. Les apothécies pourraient donc se former dans des cultures portées par un sol par lui-même défavorable à leur développement. Venant à l'appui de cette hypothèse, WiLliams \& WESTERN (1965a) notent, après expérimentation en pots placés à l'extérieur, que le trèfle violet stimule la production d'apothécies, en comparaison du sol nu et d'autres plantes.

Le sable n'est pas très favorable à la production des apothécies qui demeurent très petites. L'effet le plus spectaculaire du sable (par ses caractéristiques physico-chimiques ?) porte sur les stipes qui s'allongent (jusqu'à $30 \mathrm{~mm}$ au-dessus de la surface du sable) en direction de la lumière, sans différencier d'apothécies et finissent par se dessécher. De même, la perlite conduit à la différenciation d'un très petit nombre d'apothécies aux formes irrégulières. De nombreux stipes qui émergent n'évoluent pas, noircissent et disparaissent.

La vermiculite, dont nous nous servons habituellement, stérilisée ou non, permet une production régulière d'apothécies. Même si elle n'est pas la plus performante, elle est d'un emploi commode et s'avère la plus sûre sur une longue période.

\section{F. Action de facteurs physiques}

L'étude de l'influence de facteurs physiques porte sur la différenciation des jeunes stipes en apothécies, en 2 essais concordants, à partir de lots de 10 sclérotes de 3 à $6 \mathrm{~mm}$ de diamètre venant de produire des stipes dans les conditions optimales. Ces sclérotes sont déposés sur du coton hydrophile imbibé d'eau distillée, dans des cristallisoirs fermés par des couvercles en polystyrène transparent et placés dans les conditions à étudier. Rappelons que les conditions optimales de l'évolution normale des stipes sont une température de $15^{\circ} \mathrm{C}$, une humidité relative de $100 \mathrm{p}$. 100 et une lumière blanche de 1500 lux environ. Seul le facteur à étudier varie, les autres étant maintenus à l'optimum.

\section{Influence de la température (fig. 4 et 5)}

A $25^{\circ} \mathrm{C}$, les stipes n'évoluent pas et finissent par se nécroser, 3 semaines après le début de la manipulation. A $10{ }^{\circ} \mathrm{C}$, les stipes produisent des apothécies de petit diamètre, en faible nombre, qui se nécrosent en 10 à 15 jours. A $20^{\circ} \mathrm{C}$, la formation des apothécies est rapide, leur diamètre est assez grand, mais elles se nécrosent après 10 jours. La température la plus favorable est de $15^{\circ} \mathrm{C}$, aussi bien pour la vitesse de maturation des stipes que pour le nombre d'apothécies formées, leurs dimensions et leur persistance, puisque ces organes peuvent durer au moins 3 semaines avant de brunir et de s'affaisser.

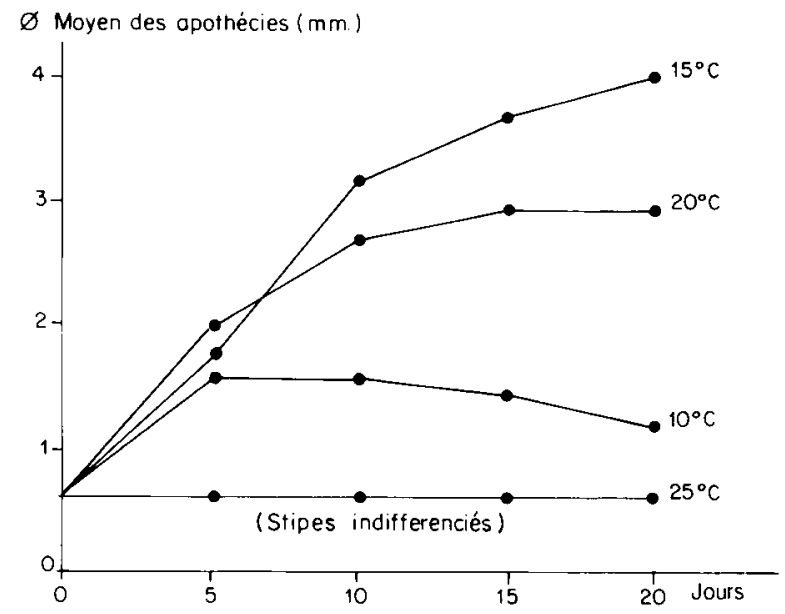

Figure 4

Action de la température sur les dimensions des apothécies en fonc tion du temps.

Action of temperature on the dimensions of apothecia in relation to time.

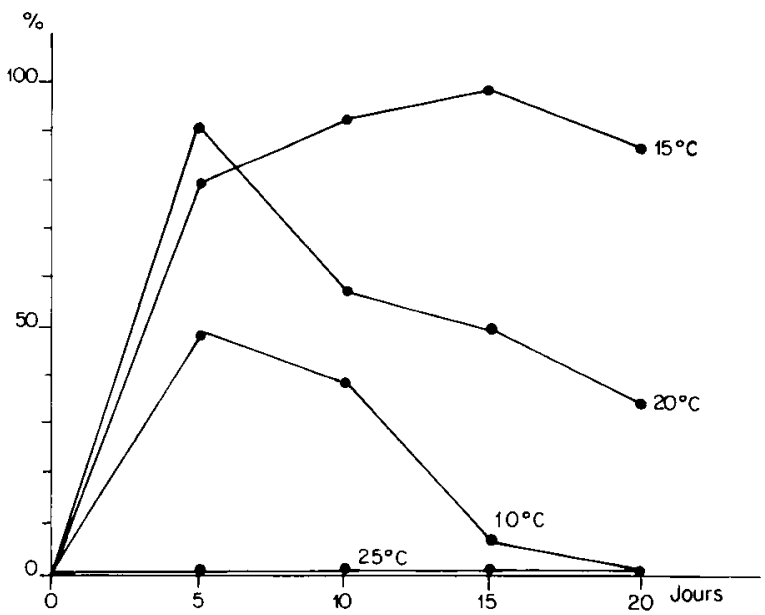

Figure 5

Action de la température sur l'évolution des stipes : pourcentages d'apothécies différenciées, non nécrosées.

Action of temperature on stipe evolution : percentages of differentiated, not necrosed apothecia.

\section{Influence de l'humidité}

Une humidité relative de l'atmosphère de 100 p. 100 et surtout une imbibition permanente des sclérotes 
sont nécessaires. Des stipes dont les sclérotes ne sont pas en contact avec le coton humide n'évoluent pas, même dans une atmosphère à 100 p. 100 d'humidité. Par contre, si les sclérotes sont en contact avec le coton humide, les stipes peuvent différencier des apothécies si l'humidité atmosphérique est supérieure ou égale à 80 p. 100. (L'humidité relative dans les cristallisoirs est réglée par des solutions salines - RAPILLY, 1968 - les sclérotes étant placés dans les fonds de petites boîtes de Petri).

\section{Influence de la lumière}

\section{a) Quantité de lumière}

Les lots de sclérotes porteurs de stipes sont soumis à des intensités lumineuses de 20,50,100,200,400, $800 \mu \mathrm{wcm}^{-2}$, en lumière blanche (tubes fluorescents " Blanc $Z$ »). Au bout de 15 jours, il n'est pas possible de différencier significativement les quantités d'apothécies produites dans les divers lots, ni leurs diamètres moyens, compris entre 3,5 et $3,9 \mathrm{~mm}$. Cependant, les stipes fortement éclairés (400 et $800 \mu \mathrm{wcm}^{-2}$ ) évoluent plus rapidement que les autres : les apothécies se forment et s'étalent en 47 jours (5-15 jours dans les autres cas), mais se nécrosent aussi plus vite après avoir sporulé (80 p. 100 des apothécies sont affaissées et en voie de nécrose au $15^{\mathrm{e}}$ jour, alors que les autres sont turgescentes).

L'effet d'un éclairement continu ne peut être différencié d'une photopériode de $12 \mathrm{~h} / 24$, pour une même intensité lumineuse $\left(200 \mu \mathrm{wcm}^{-2}\right)$, tant en ce qui concerne la rapidité d'évolution des stipes que les nombres et les dimensions des apothécies formées. Par contre, l'obscurité continue empêche les stipes de se transformer. Reportés à la lumière, ils donnent dans quelques cas des apothécies normales, mais le plus souvent demeurent stériles ou parfois forment de multiples petites apothécies à leur sommet (fig. 6).

\section{b) Qualité de la lumière}

Des lots de sclérotes porteurs de stipes soumis à une intensité lumineuse identique $\left(160 \mu \mathrm{wcm}^{-2}\right)$, sont

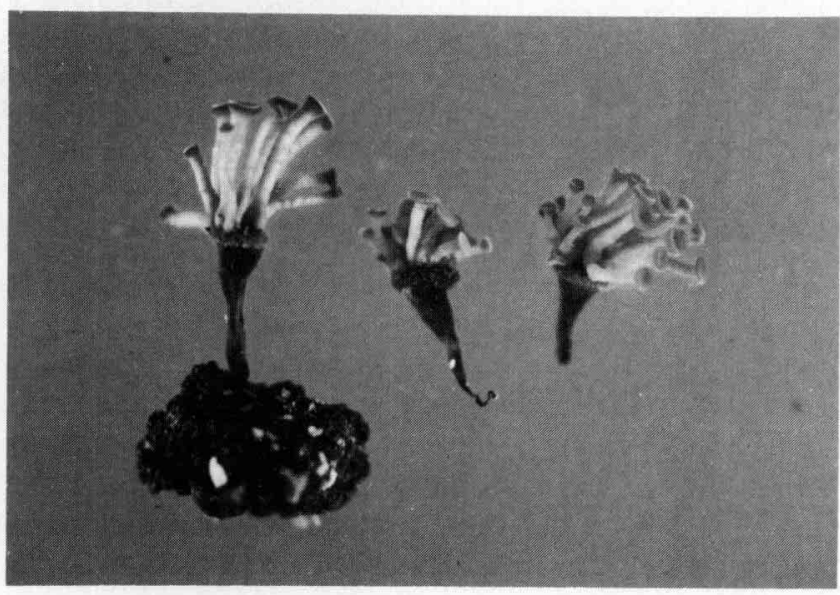

Figure 6

Apothécies multiples différenciées par des ébauches d'apothécies ayant repris leur croissance après un arrêt momentané.

Multiple apothecia differentiated from aborted ones which resumed their growth after a temporary stop. exposés à la lumière blanche (tubes fluorescents " Blanc $Z$ ») ou à des lumières colorées (dont le domaine spectral est mesuré) obtenues en interposant des feuilles de rhodoïd de couleur entre la source lumineuse blanche et le champignon.

Le tableau 7 montre que la différenciation des stipes nécessite, pour être la plus complète, la totalité du spectre lumineux. La lumière verte est plus efficace que les autres, notamment la bleue dont l'action inductrice des fructifications est connue chez de nombreux champignons (DURAND, 1976; DELMAS \& MAMOUN, 1982). S. trifoliorum semble marquer en fait d'après nos expérimentations une photosensibilité assez peu spécifique, avec toutefois une meilleure carpogenèse sous des radiations de grandes longueurs d'onde.

\section{TABLEAU 7}

Influence de la longueur d'onde de la lumière sur la différenciation des stipes en apothécies.

Influence of the wavelength of light on stipe differentiation in apothecia.

\begin{tabular}{ccccc}
$\begin{array}{c}\text { Couleur de } \\
\text { la lumière }\end{array}$ & $\begin{array}{c}\text { Domaine } \\
\text { spectral } \\
(\mathrm{nm})\end{array}$ & $\begin{array}{c}\text { Pic de } \\
\text { radiations d'apothécies } \\
(\mathrm{nm})\end{array}$ & $\begin{array}{c}\text { Pourcentage } \\
\text { évoluées }\end{array}$ & $\begin{array}{c}\text { Diamètre moyen } \\
\text { après 15 jours } \\
\text { apm) }\end{array}$ \\
\hline blanche & $380-750$ & $550-650$ & 100 & 3,7 \\
bleue & $400-575$ & $475-500$ & 10 & 1,3 \\
verte & $450-625$ & $525-550$ & 30 & 3,1 \\
jaune & $475-725$ & $575-600$ & 20 & 1,8 \\
rouge & $575-750$ & $600-625$ & 20 & 2,0 \\
\hline
\end{tabular}

\section{G. Influence de l'isolat de $S$. trifoliorum}

L'isolat de trèfle violet que nous utilisons habituellement pour la production d'apothécies, «ST.78.02», a été le premier avec lequel nous avons travaillé à ce sujet et nous sert donc de référence. En 1985-86, nous avons comparé 8 isolats de trèfle violet et 11 de luzerne provenant de 12 départements de la moitié nord de la France, à «ST.78.02 », dans les conditions énoncées au chapitre II. L'essai est répété 2 fois.

La variabilité entre les 2 essais est forte, sauf pour ST.78.02, dont la production est relativement constante. Il n'est donc pas possible de présenter des résultats chiffrés sur ces 2 essais. Tout au plus peut-on dégager des tendances. Tout d'abord, un nombre non négligeable d'isolats ( 3 sur luzerne et 2 sur trèfle) refusent de former des apothécies dans nos conditions, bien qu'ils différencient des sclérotes apparemment normaux. Parmi ces isolats, 2 ont été conservés longtemps in vitro (depuis 1973 et 1974), mais les autres ont été isolés récemment (1980 et 1984). Le passage in vitro pendant une longue période n'empêche pas obligatoirement la différenciation des apothécies, cependant les isolats les plus productifs sont ceux qui ont été isolés à partir de 1980 . Le plus productif reste ST.78.02, fréquemment repiqué à partir de réisolements d'ascospores. Il n'est donc pas impossible que l'on ait ainsi sélectionné son aptitude à la carpogenèse. Enfin, il n'existe pas de différence nette de productivité ni de dimensions d'apothécies, ni de quantités d'ascospores par apothécies, entre les isolats de trèfle et de luzerne. 
Notons en outre qu'un isolat de $S$. sclerotiorum de carottes a produit dans les mêmes conditions d'abondantes apothécies (environ 2 fois plus que ST.78.02) indifférenciables par leur aspect de celles de nos isolats de $S$. trifoliorum. Dans cet essai en chambre climatisée, $S$. sclerotiorum a produit des apothécies de la mi-octobre à décembre 1983, alors que dans la nature, les apothécies se forment hors de la saison froide. Si cela se vérifiait, il serait donc possible de produire des apothécies de $S$. sclerotiorum de la même façon que pour $S$. trifoliorum, quelle que soit la saison.

\section{H. Anomalies de la carpogenèse}

Lorsque pour des raisons diverses (température trop élevée, humidité trop faible, panne d'éclairage,...) les apothécies sont momentanément gênées dans leur développement, il est assez fréquent qu'elles soient atteintes d'anomalies. On voit par exemple des apothécies à l'hyménium festonné ou plus ou moins coralliforme qui produisent tout de même des quantités appréciables d'ascospores. Dans certains cas, lorsque la différenciation des stipes est interrompue, puis reprend au retour des conditions favorables, leur extrémité s'orne de multiples stipes secondaires qui mûrissent en petites apothécies (fig. 6). Notons que certains isolats semblent plus sujets que d'autres à ces anomalies, notamment que ST.78.02. Ces observations recoupent celles faites sur $S$. sclerotiorum par CASALE \& HART (1986), ainsi que RADKE \& GRAU (1986) sur des sclérotes ayant reçu des traitements herbicides, de même que celles de KOSASIH et WILLETTS (1975) sur des isolats de la même espèce ayant subi des fluctuations thermiques et lumineuses ou chez lesquels on a intentionnellement décapité les stipes. La régulation de la morphogenèse des apothécies, bien que mal comprise, semble donc comparable chez $S$. trifoliorum et $S$. sclerotiorum.

\section{CONCLUSION}

L'étude de la carpogenèse des Sclerotinia a fait l'objet d'un nombre de travaux relativement réduit. L'espèce la plus étudiée a été $S$. sclerotiorum, en raison de sa vaste répartition géographique, de l'importance économique qu'elle présente à cause de son caractère polyphage et par le fait que ses sclérotes acceptent apparemment assez facilement de former des apothécies au laboratoire. Très peu de travaux, par contre, ont porté sur $S$. trifoliorum, souvent considéré comme inconstant dans sa carpogenèse lorsqu'il est hors des conditions naturelles. En réalité, il apparaît, d'après nos travaux sur la carpogenèse de cette espèce, de grandes similitudes avec $S$. sclerotiorum en ce qui concerne le déterminisme de la formation des apothécies, ce qui confirme la proximité taxonomique de ces deux espèces, proximité qui a pu entraîner quelques incertitudes, confusions, voire erreurs manifestes de détermination. Ces similitudes exigent nous semblet-il que nous légitimions l'idendité des isolats ayant servi à nos travaux, par une mise au point comparative sur les critères de différenciation des deux Sclerotinia.
Le fait que le champignon soit isolé sur trèfle ou luzerne ne signifie en effet pas à coup sûr que l'on soit en présence de $S$. trifoliorum, puisque $S$. sclerotiorum est susceptible d'attaquer ces cultures parmi les nombreux végétaux de sa gamme d'hôtes. Cependant, S. trifoliorum est considéré par la majorité des auteurs comme spécialisé aux légumineuses fourragères et nous pensons pour notre part, ainsi que d'autres, que les dégâts sur trèfles et luzerne sont surtout le fait de $S$. trifoliorum dont les caractéristiques lui permettent de se développer pendant la saison froide pour aboutir aux pourritures printanières fréquemment graves. Le fait que $S$. trifoliorum différencie ses apothécies exclusivement en automne le rend par ailleurs pratiquement inapte à attaquer les autres cultures sur lesquelles sévit $S$. sclerotiorum. A l'inverse, il est fort peu probable que $S$. sclerotiorum aboutisse aux pourritures que l'on constate au printemps sur les légumineuses fourragères, étant donné ses exigences en température et sa période de carpogenèse différentes de celles de $S$. trifoliorum. Par contre, il n'est pas exclu que $S$. sclerotiorum provoque quelques pourritures d'importance secondaire pendant la bonne saison. Les isolats utilisés pour nos manipulations ont donc de très grandes chances de bien appartenir à l'espèce trifoliorum, car tous ont été isolés de trèfle ou de luzerne, soit en fin d'hiver - début de printemps à partir d'ébauches de sclérotes, soit à partir d'apothécies formées en automne (cas de notre isolat de référence ST.78.02).

Une autre cause d'incertitude est le fait qu'aucun caractère morphologique macroscopique ne permette de différencier sûrement les deux espèces. Les sclérotes, le mycélium, de même que les apothécies sont très semblables. Cependant, dans le cas de $S$. trifoliorum, ainsi que nous l'avons vérifié pour notre isolat de référence, les asques montrent un dimorphisme des ascospores ( 4 petites et 4 légèrement plus volumineuses), ce qui n'existe pas chez $S$. sclerotiorum (KOHN, 1979). De plus, les caractères de croissance mycélienne en fonction de la température permettraient de distinguer les deux espèces. Ainsi, en moyenne, la température optimale pour la croissance des isolats de $S$. sclerotiorum est voisine de $25^{\circ} \mathrm{C}$ alors que les isolats de $S$. trifoliorum sont favorisés entre 15 et $20^{\circ} \mathrm{C}$, ce que nous avons vérifié pour quelques-uns de nos isolats (WILlis, 1970). Cependant, des isolats "froids " de $S$. sclerotiorum pourraient par ce critère être confondus avec ceux de $S$. trifoliorum. D'autre part, la confrontation in vitro de cultures mycéliennes des deux espèces donne lieu à un phénomène de "barrage », qui n'a pas lieu lorsque 2 cultures d'une même espèce sont confrontées (WONG \& WILLETS, 1975). C'est ainsi que nos isolats de trèfle et de luzerne, confrontés avec un isolat identifié comme étant S. sclerotiorum, manifestent de façon le plus souvent assez nette ce phénomène qui se traduit par une zone brune de quelques millimètres séparant les colonies. Enfin, l'analyse électrophorétique des estérases permet de séparer de façon plus précise ces deux Sclerotinia, de même que $S$. minor (SCOTT, 1981). Les faits plaident donc pour une réelle distinction des deux espèces.

Afin de lever toute ambiguïté, il conviendrait néanmoins de pouvoir différencier ces deux Sclerotinia avec encore plus de certitude, pour des raisons taxonomiques, épidémiologiques, mais aussi pratiques. Il 
se pose en effet souvent la question du type de culture (par exemple tournesol) à implanter après une légumineuse fourragère détruite au printemps par la sclérotiniose. Tant que subsistera un doute dans l'identification de l'espèce responsable des dégâts, on ne pourra pas donner de réponse sans équivoque sur le risque encouru par la culture de remplacement ou qui suit la légumineuse dans la rotation. D'autre part, une identification précise des isolats est nécessaire pour la sélection de la résistance.

Nos travaux sur la carpogenèse de $S$. trifoliorum permettent par ailleurs d'obtenir déjà régulièrement de grandes quantités d'apothécies quelle que soit la saison, car toutes les étapes, depuis l'obtention des sclérotes jusqu'à la différenciation des apothécies, peuvent se dérouler en chambre climatisée. Cependant, de nombreux points resteraient à préciser, notamment les mécanismes d'initiation et de levée de dormance des sclérotes. Ceci permettrait peut-être d'augimenter le rendement en apothécies et de comprendre pourquoi certains isolats n'ont pas différencié d'apothécies dans nos conditions. De même, la technique utilisée pour la récolte des ascospores ne permet en aucune façon de connaître la cinétique et l'intensité de la sporulation des apothécies. Cette étape très importante de la biologie de $S$. trifoliorum et pour la compréhension de l'épidémiologie de la sclérotiniose, est pour l'instant inconnue. La possibilité que nous avons de produire à tout moment des apothécies au laboratoire nous permet de l'étudier facilement en fonction de divers facteurs, ce que nous faisons actuellement.

Enfin, les grandes quantités d'ascospores produites au cours des diverses manipulations ont été utilisées pour mettre au point une technique de sélection du trèfle violet pour la résistance à la sclérotiniose. Nous en rapporterons les principaux résultats dans un prochain article. L'inoculum obtenu en chambre de culture, de même que la technique d'inoculation sont désormais utilisés par les sélectionneurs.

Reçu le 6 avril 1987. Accepté le 8 juillet 1987.

\section{RÉFÉRENCES BIBLIOGRAPHIQUES}

Casale W. L., Hart L. P., 1986. Influence of four herbicides on carpogenic germination and a pothecium development of Sclerotinia sclerotiorum. Phytopathology, 76, 980-984.

Delmas J., Mamoun M., 1982. Influence de la lumière sur la fructification in vitro du pleurote corne d'abondance, Pleurotus cornupiae Fr. ex P. Agronomie, 2, 379-388.

Dijkstra J., 1966a. Acceleration of apothecia development of Sclerotinia trifoliorum Neth. J. Pl. Path., 72, 203.

Dijkstra J., $1966 b$. Rotting by spread of mycelium from ascospore lesions of Sclerotinia trifoliorum Neth. J. Pl. Path., 72, 279-283.

Durand R., 1976. Influence des radiations lumineuses sur les processus de reproduction des champignons; hypothèses sur l'identité des photorécepteurs. Revue bibliographique. Mycopathologia, 60, 3-16.

McGimpsey H. C., Malone J. P., 1979. Production of apothecia of Sclerotinia trifoliorum in the laboratory. Bull. Br. mycol. Soc., 13, 104.

Kohn L. M., 1979. A monographic revision of the genus Sclerotinia. Mycotaxon, 9, 365-444.

Kosasih B. D., Willetts H. J., 1975. Types of abnormal apothecia produced by Sclerotinia sclerotiorum. Mycologia, 67, 89-97.

Iamarque C., 1980. Obtention d'ascospores de Sclerotinia sclerotiorum (Lib.) De Bary et techniques d'inoculation utilisables dans la sélection variétale du tournesol. Inf. tech. CETIOM, 71, 22-27.

Loveless A. R., 1951. Observations on the biology of clover rot. Ann. appl. Biol., 38, 642-665.

Radke V. L., Grau C. R., 1986. Effect of herbicides on carpogenic germination of Sclerotinia sclerotiorum. Plant Disease, 70, 19-23.

Rapilly F., 1968. Les techniques de mycologie en pathologie végétale. Ann. Epiphyties, 19, hors série.

Raynal G., 1981. La sclérotiniose des trèfles et des luzernes à Sclerotinia trifoliorum Eriks. I. Choix d'une méthode de contamination artificielle. Agronomie, 1, 565-572.
Raynal G., 1983. Production au laboratoire d'apothécies de Sclerotinia trifoliorum Eriks. pour l'évaluation de la résistance du trèfle violet à la sclérotiniose. Agronomie, 3, 369-373.

Raynal G., 1984. Observations sur la formation des apothécies de Sclerotinia trifoliorum Eriks. à partir de sclérotes produits au laboratoire. 25 Coll. Soc. Fr. Phytopath., Agronomie, 4, 696.

Raynal G., 1985. Observations sur le comportement variétal au champ du trèfle violet vis-à-vis de la sclérotiniose et sur l'épidémiologie de la maladie. Fourrages, 101, 85-104.

Schwartz H., Steadman J. R., 1978. Factors affecting sclerotium populations of, and apothecium production by, Sclerotinia sclerotiorum. Phytopathology, 68, 383-388.

Scott S. W., 1981. Separation of Sclerotinia isolates collected from three herbage legume hosts. Trans. Br. mycol. Soc., 76, 321-323.

Tourvielle de Labrouhe D. et al., 1978. Rôle des ascospores dans l'infection du tournesol par Sclerotinia sclerotiorum (Lib.) De Bary. Ann. Phytopathol., 10, 417-431.

Williams G. H., Western J. H., 1965a. The biology of Sclerotinia trifoliorum Eriks. and other species of sclerotium-forming fungi. I. Apothecium formation from sclerotia. Ann. appl. Biol., 56, 253260.

Williams G. H., Western J. H., 1965b. The biology of Sclerotinia trifoliorum Eriks. and other species of sclerotium-forming fungi. II. The survival of sclerotia in soil. Ann. appl. Biol., 56, 261-268.

Willis C. B., 1970. Incubation temperature differentiates Sclerotinia trifoliorum from S. sclerotiorum. Proc. Can. Phytopathol. Soc., 37, 29.

Wong A. L., Willetts H. J., 1975. A taxonomic study of Sclerotinia sclerotiorum and related species : mycelial interactions. Journal of General Microbiology, 88, 339-344. 